\title{
Nogo-B promotes epithelial-mesenchymal transition in lung fibrosis via PERK branch of the endoplasmic reticulum stress pathway
}

\author{
Ying Zhu ${ }^{1 \#}$, Meng Yang ${ }^{2 \#}$, Xue-Hui Li ${ }^{1 \#}$, Wu-Jian $\mathrm{Xu}^{3}$, Wei Gao ${ }^{3}$, Yu-Han Chen ${ }^{4}$, Jian-Dong Li ${ }^{1}, \mathrm{Qiang} \mathrm{Li}^{3}$ \\ ${ }^{1}$ Department of Respiratory and Critical Care Medicine, Seventh Medical Center of Chinese PLA General Hospital, Beijing, China; ${ }^{2}$ Department \\ of Geriatrics, Changhai Hospital, Navy Military Medical University, Shanghai, China; ${ }^{3}$ Department of Respiratory and Critical Care Medicine, \\ Shanghai East Hospital, Tongji University School of Medical, Shanghai, China; ${ }^{4}$ National Engineering Laboratory for Birth Defects Prevention \\ and Control of Key Technology, Beijing Key Laboratory of Pediatric Organ Failure, Affiliated Bayi Children's Hospital, Seventh Medical Center of \\ Chinese PLA General Hospital, Beijing, China \\ Contributions: (I) Conception and design: Y Zhu, JD Li, Q Li; (II) Administrative support: JD Li, Q Li; (III) Provision of study materials or patients: \\ Y Zhu, M Yang; (IV) Collection and assembly of data: Y Zhu, XH Li, WJ Xu; (V) Data analysis and interpretation: Y Zhu, W Gao, YH Chen; (VI) \\ Manuscript writing: All authors; (VII) Final approval of manuscript: All authors. \\ "These authors contributed equally to this work. \\ Correspondence to: Jian-Dong Li. Department of Respiratory and Critical Care Medicine, Seventh Medical Center of Chinese PLA General Hospital, \\ 5 Nanmencang, Dongcheng District, Beijing 100700, China. Email: lijdzyy@163.com; Qiang Li. Department of Respiratory and Critical Care \\ Medicine, Shanghai East Hospital, Tongii University School of Medical, Shanghai 200120, China. Email: Liqres@163.com.
}

Background: Idiopathic pulmonary fibrosis (IPF) is a fatal chronic pulmonary fibrosis disease and pathological mechanisms of fibrogenesis in IPF are still to be elucidated. Here, we investigated the potential role of Nogo-B in pulmonary fibrogenesis.

Methods: A mouse model of pulmonary fibrosis was established by intratracheal injection of bleomycin (BLM). Lung epithelial cells MLE-12 and TC-1 JHU-1 were cultured for TGF- $\beta$ treatment. The extent of lung fibrosis was evaluated using hematoxylin and eosin (HE) staining and Masson staining in model mice and Nogo-B knockout mice. The protein levels of Nogo-B, endoplasmic reticulum stress (ERS) sensors including PERK, IRE1 $\alpha$, ATF6 and epithelial-mesenchymal transition (EMT) markers including E-cadherin and $\mathrm{N}$-cadherin, vimentin were assayed by Western blotting respectively after Nogo-B knockdown or overexpression with lentivirus. Enzyme-linked immunosorbent assay (ELISA) was used to evaluate cytokine levels of TGF- $\beta$, TNF- $\alpha$, IL-1 $\beta$, IL-6 and IL-10 in bronchoalveolar lavage fluid (BALF).

Results: Nogo-B expression was up-regulated in lung tissues of fibrosis model mice and alveolar epithelial cells. Nogo-B knockdown significantly attenuated lung fibrogenesis, downregulated the levels of inflammatory cytokines, inhibited EMT as well as decreased the level of phosphor-PERK/PERK but not the levels of phosphor-IRE1 $\alpha /$ IRE1 $\alpha$ and c-ATF6. Additionally, a potential efficacy of PERK blockade was demonstrated in improving the extent of lung fibrosis in model mice.

Conclusions: This study discovered that involvement of Nogo-B in pulmonary fibrogenesis was associated with the PERK branch of ERS pathway and EMT. Nogo-B could be considered as a potential therapeutic target for the treatment of IPF.

Keywords: Idiopathic pulmonary fibrosis (IPF); Nogo-B; epithelial-mesenchymal transition (EMT); endoplasmic reticulum stress (ERS); PERK

Submitted Aug 27, 2020. Accepted for publication Jan 05, 2021.

doi: $10.21037 / \mathrm{atm}-20-6143$

View this article at: http://dx.doi.org/10.21037/atm-20-6143 


\section{Introduction}

Idiopathic pulmonary fibrosis (IPF) is an irreversible, progressive, and fatal chronic pulmonary fibrosis disease, characterized by diffuse pulmonary interstitial fibrosis with mild inflammation, appearance of fibroblastic foci and deposition of matrix (1). Incidence of IPF has risen over time and is increasingly recognized (2). Recently, increasing evidence indicates that abnormal extracellular matrix deposition contributes to disease pathogenesis and genetic susceptibility plays a role in the development of IPF (3), but molecular mechanism of IPF progression is poorly understood. Hence, unravelling the pathological mechanisms of fibrogenesis in IPF is still challenging.

The formation of lung fibrosis is complex, resulting from a persistent irritant that sustains the production of growth factors, proteolytic enzymes, angiogenic factors and fibrogenic cytokines; these factors stimulate the deposition of connective tissue elements that progressively remodel and destroy normal tissue architecture (4). Recently, studies show that epithelial-mesenchymal transition (EMT) is a key step of pulmonary fibrosis; meanwhile, some key signaling pathways and microRNAs are involved in $\operatorname{EMT}(5,6)$. Thus, understanding fully the role of EMT in the development of pulmonary fibrosis will be conducive in search for new methods and new drugs for the treatment of IPF.

Of note, more recently, studies have found that the activation of endoplasmic reticulum stress (ERS) in alveolar epithelial cells of patients with IPF is significantly enhanced (7), suggesting that ERS may play an important role in the development of IPF. As a member of the reticulon protein family, Nogo-B, also known reticulon 4B (RTN4B), is widely expressed in blood vessels, lung, kidney and has very important physiological functions (8). Functionally, it can regulate growth of vascular endothelial cells, induce apoptosis of tumor cells and modulate differentiation of immune-inflammatory cells $(9,10)$ and can also participate in tissue repair through TGF- $\beta / \mathrm{Smad} 2$ signaling pathway (11). Until now, the roles of Nogo-B in hepatic fibrosis are well investigated and it can serve as a regulator in hepatic stellate cell activation (12). These findings encourage us to hypothesize that Nogo-B may also be involved in the pathogenesis of IPF (13). In the study, we verified that Nogo-B was significantly upregulated in IPF model mice and served as an important mediator for lung fibrogenesis. Mechanistically, Nogo-B deficiency downregulated the levels of inflammatory cytokines, inhibited EMT as well as decreased the activity of the PERK branch of ERS pathway.
This study delineated a previously unidentified pathway, in which Nogo-B sensed PERK pathway and facilitated the EMT of pulmonary epithelial cells, finally promoting the lung fibrosis in IPF. We present the following article in accordance with the ARRIVE reporting checklist (available at http://dx.doi.org/10.21037/atm-20-6143).

\section{Methods}

\section{Animals and experimental procedures}

Firstly, a mouse model of BLM-induced pulmonary fibrosis was established according to previous study (14). In brief, all of 40 C57BL/6 mice [male, 7-8 weeks old, 18-20 g weight; specific pathogen free (SPF)] were fasted in an airconditioned room with $12 \mathrm{~h}$ light and dark cycles and had free access to water on 1 day before modeling; then were anesthetized by intraperitoneal injection of $3 \%$ sodium pentobarbital at a dose of $0.13 \mathrm{~mL} / 100 \mathrm{~g}$ on the day of modeling. After randomization, 30 mice were instilled with bleomycin (BLM) $(10 \mathrm{mg} / \mathrm{kg})$ into oropharyngeal cavity after the tongues of anesthetized mice at one time to establish an animal model of pulmonary fibrosis; the other 10 control mice were instilled with an equal volume of $0.9 \% \mathrm{NaCl}$ into the trachea. For the model group, 10 mice were randomly chosen for treatment with PERK blockade (ISRIB; $0.25 \mathrm{mg} / \mathrm{kg}$ ) and 10 mice were designed as control. Lung tissue and BALF were collected for immunohistochemistry (IHC) analysis and detection of molecular markers. Moreover, 2-month-old male Nogo-B $\mathrm{KO}\left(\right.$ Nogo-A/B $\left.{ }^{--}\right)$and age-matched wild-type C57BL/6 mice were obtained from Shanghai East Hospital, Tongji University School of Medicine. Experiments were performed under a project license (No. 2020-097) granted by the Ethics Committee of Seven Medical Center of Chinese PLA General Hospital, in compliance with institutional guidelines for the care and use of animals.

\section{Hematoxylin and eosin (HE) staining and Masson staining}

Before staining, tissue slides were dried in a $65^{\circ} \mathrm{C}$ incubator for 1 hour. Hematoxylin-eosin staining and Masson staining were performed according to the standard protocol; slides were examined by light microscopy and photographed for evaluation of histopathology and collagen deposition, respectively. Histopathological scoring of pulmonary fibrosis was performed according to the method reported by previous study (15). 


\section{IHC}

Lung samples were fixed in formalin solution and embedded by paraffin. These formalin-fixed paraffin-embedded (FFPE) sections were used for IHC staining with primary antibody against Nogo-B. Sections were deparaffinized and permeabilized in citrate buffer solution by microwaving for proteolysis and then washed in phosphate buffer solution (PBS) and endogenous peroxidase activities were blocked by $3 \% \mathrm{H}_{2} \mathrm{O}_{2}$ for 15 min. After washing with $\mathrm{PBS}$ three times, the sections were blocked with goat serum for $30 \mathrm{~min}$, followed by incubation with primary antibody against the Nogo-B (1:200; AF6034, R\&D system) at $4{ }^{\circ} \mathrm{C}$, overnight. The subsequent steps were performed following the instructions of the secondary biotinylated antibody kit (Zhongshan Biotech, Beijing, China). Histological pictures were taken at 200x magnification by using Nikon digitalsight imaging system attached to Nikon E-600 microscope (Nikon Corporation, Japan).

\section{Bronchial alveolar lavage fluid (BALF) analysis}

Briefly, bronchoalveolar lavage (BAL) of the mice was performed four times each with $0.5 \mathrm{~mL}$ of saline through the trachea and was consistently recovered with gentle handling. The suspension was centrifuged at $1,500 \mathrm{rpm}$ for 5 minutes at $4{ }^{\circ} \mathrm{C}$; then, the supernatant of BAL obtained from $2 \mathrm{~mL}$ of instilled saline was stored at $-20{ }^{\circ} \mathrm{C}$ until carrying out an enzyme linked immunosorbent assay (ELISA) assay for measurements of cytokines (TGF- $\beta$, TNF- $\alpha$, IL- $1 \beta$, IL- 6, IL-10) in the BALF.

\section{Cell culture and primary cell isolation}

Mouse lung epithelial cells (MLE-12 and TC-1 JHU-1) were purchased from the American Type Culture Collection (Manassas, VA, USA) and cultured respectively with DMEM/F-12 (Gibco, Waltham, MA, USA) containing $2 \%$ fetal bovine serum (FBS) (Gibco) and RPMI-1640 containing $10 \% \mathrm{FBS}$ at $37^{\circ} \mathrm{C}$ in a humidified atmosphere containing 5\% CO2 and $95 \%$ air. The cells were maintained at sub-confluent densities and used when growing to the third passages.

Primary type II alveolar epithelial cells (AECII) were isolated from $\mathrm{C} 57 \mathrm{BL} / 6$ mice according to previous description (16). In brief, the lungs were perfused with Hank's Balanced Salt Solution (HBSS) and incubated in diapase-containing solution for $45 \mathrm{~min}$; then were cut into pieces for subsequent digestion with DNAse I (Thermo Scientific). After filtering through 70 and $40 \mu \mathrm{m}$ nylon cell strainers (BD Falcon, USA), the cells were centrifuged at $800 \mathrm{rpm}$ for $10 \mathrm{~min}$. Then cell suspensions were incubated in tissue cultured plates at $37^{\circ} \mathrm{C}$ for about $6 \mathrm{~h}$, and nonattached cells were centrifuged again and were enumerated. Finally, AECs were cultured on Matrigel-coated plates (BD Biosciences) for further experiments.

\section{Lentivirus-mediated gene knockdown and overexpression}

The short hairpin RNA (shRNA) carrier was constructed to target Rtn4 gene or perk gene by inserting respectively three different target sequences into the plasmid pLKO.1. In brief, lentiviral vectors pLKO.1 TRC and pWPI.1 were used for constructing recombinant lentiviruses of short interference RNA (shRNA) constructs and Nogo-B or PERK shRNA, non-targeting shRNA (shNT). Recombinant lentivirus was amplified in HEK293T cells. The lentivirus for overexpressing Nogo-B (OV-Nogo-B) and the control lentivirus were obtained commercially from GenePharma Corporation (Shanghai, China). After transfection, MLE-12 and TC-1 JHU-1 cells with stable Nogo-B or PERK knockdown or Nogo-B overexpression were screened and obtained by puromycin for subsequent detection with real-time PCR and Western blotting.

\section{Real-time PCR}

Real-time PCR was analyzed by LightCycler ${ }^{\circledR} 480$ RealTime PCR system (Roche, Switzerland) with reagent of SYBR PrimeScriptTM RT Reagent Kit (TaKaRa, Japan). The assay was performed according to the manufacturer's protocol.

\section{Western blotting}

The protein concentration was determined using the BioRad protein assay system. Cells were plated at $6 \times 10^{5}$ cells per well in six-well plates. After treatment, cells were washed with lysed using RIPA lysis buffer containing $1 \mathrm{mM}$ phenylmethylsulfonyl fluoride (PMSF). Twenty $\mu \mathrm{g}$ proteins underwent electrophoresis on a $10 \%$ or $8 \%$ SDSpolyacrylamide gel; proteins separated in the gel were transferred to a $0.2 \mathrm{~mm}$ polyvinylidene fluoride membrane (PVDF, Millipore, Billerica, USA) using a Bio-Rad semidry instrument. After blocking with $5 \%$ BSA in TBS 
containing $0.1 \%$ Tween-20 for $1 \mathrm{~h}$ at room temperature, the membranes were incubated with a primary antibody including sheep anti-mouse Nogo-B (1:1,000; AF6034), rabbit anti-mouse total or phospho-PERK (all, 1:1,000; \#3192 and \#3179), rabbit anti-mouse total or phosphoIRE1 $\alpha$ (1:1,000; \#3294 and PA1-16927), rabbit antimouse c-ATF6 (1:1,000; ab203119), rabbit anti-mouse ZO-1 (1:1,000; ab96587), rabbit anti-mouse SPC (1:1,000; ab196677), rabbit anti-mouse $\alpha$-SMA (1:1,000; ab108424), rabbit anti-mouse MMP4 (1:500; ab51074), sheep antimouse E-cadherin (1:1,000; sc-1500), rabbit anti-mouse $\mathrm{N}$-cadherin (1:1,000; SC-7939) or rabbit anti-mouse vimentin $(1: 1,000$; sc-5565) respectively from $R \& D$ system, Cell Signaling Technology Company, Thermo Scientific ${ }^{\mathrm{TM}}$, Abcam and Santa Cruz Biotechnology Company, overnight at $4^{\circ} \mathrm{C}$. $\beta$-actin was regarded as the internal reference, diluted in TBST with 1:2,000. After washing, the membranes were incubated with an anti-rabbit or anti-sheep secondary antibody and performed using the ECL western blot system (Super Signal West Pico Chemiluminescent Substrate, Pierce, Rockford, IL, USA) according to the manufacturer's instruction. Quantification of western blots was analyzed densitometrically using QuantityOne software (Bio-Rad Laboratories, Inc., Hercules, CA, USA).

\section{Migration assay}

The migration assay was performed using Transwell plates (Costar, USA). During this assay, lung epithelial cells $\left(5 \times 10^{4}\right.$ in $100 \mu \mathrm{L}$ serum-free medium) were placed on the upper layer of a cell culture insert with permeable membrane and a complete medium was placed below the cell permeable membrane. The chamber was incubated in a humidified environment with $5 \% \mathrm{CO}_{2}$ at $37{ }^{\circ} \mathrm{C}$ for $24 \mathrm{~h}$. Cells that had not migrated through the membrane were removed from the upper surface with a cotton swab; then migrated cells on the lower membrane surface were fixed and stained with crystal violet; then counted and photographed in five random fields.

\section{Statistical analysis}

The experimental data were statistically analyzed by statistical analysis software (SPSS 19.0, Chicago, IL, USA) and statistical mapping was performed by GraphPad Prism 7.0. The normal distribution of data was firstly identified by using the Kolmogorov-Smirnov test. Then, for quantitative variables with normal distribution, comparison among them was performed by using one-way analysis of variance (ANOVA) and post hoc Tukey test; for quantitative variables without normal distribution, the non-parametric test (Kruskal-Wallis) was used. The qualitative variables using the Chi-square test and the Student's $t$-test. The association between the various factors was determined using Pearson's correlation. There was a statistically significant difference in data at $\mathrm{P}<0.05$.

\section{Results}

\section{Nogo-B was up-regulated in lung tissues of fibrosis model mice and alveolar epithelial cells}

To elucidate the role of Nogo-B in the progression of pulmonary fibrosis, we firstly established a mouse model of BLM-mediated pulmonary fibrosis, and investigated the expression of Nogo-B in lung tissues. As illustrated in Figure $1 \mathrm{~A}$, compared with normal control group, model group showed that the lung tissue structure was damaged, and the alveolar interval was widened, infiltrated inflammatory cells including neutrophils, lymphocytes, and eosinophils were increased, mainly in the interalveolar septa regions. The result of Masson staining showed that the lung tissue structure was basically normal, and a small amount of blue collagen fibers were seen on the bronchial wall and blood vessel wall in normal control group; while in model group, obviously increased blue collagen fibers were flocculated and deposited in the widened alveolar interval, the terminal trachea and around the blood vessels. Meanwhile, levels of the cytokine TGF- $\beta$ and pro-inflammatory cytokines including TNF- $\alpha$, IL- $1 \beta$, IL-6 were significantly increased (all $\mathrm{P}<0.01$ ) while levels of anti-inflammatory factor IL-10 went down $(\mathrm{P}<0.05)$ in BALF (Figure $1 B)$.

To determine whether Nogo-B expression was associated with pulmonary fibrosis, we examined the expression of Nogo-B protein in lung samples of mice between the model group and the normal control group by using IHC assay and found a significantly stronger brown staining for Nogo-B in the lung mesenchyme of model group than that in its counterpart (Figure 1A). Furthermore, AECII in lung tissue of model mice on the $7^{\text {th }}$ and $28^{\text {th }}$ day were isolated and purified; moreover, expressions of Nogo-B, E-cadherin and $\mathrm{N}$-cadherin, vimentin were detected by western blotting. The result showed that E-cadherin expression was significantly decreased and expressions of Nogo-B and $\mathrm{N}$-cadherin, vimentin were significantly elevated (Figure 1C,D). Taken together, Nogo-B was involved in the 


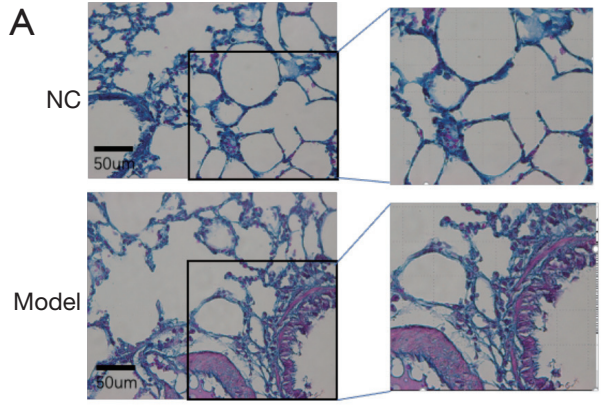

Masson staining

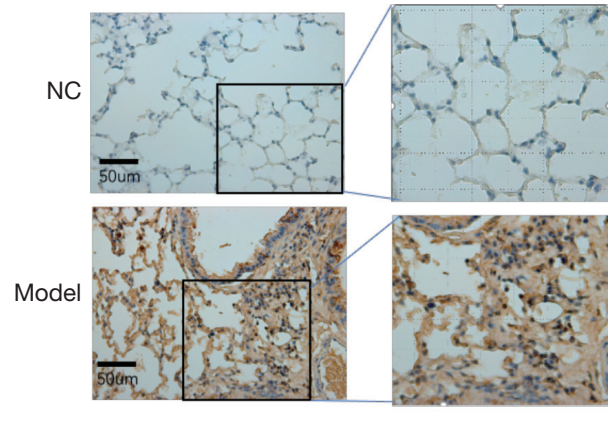

IHC with Nogo-B
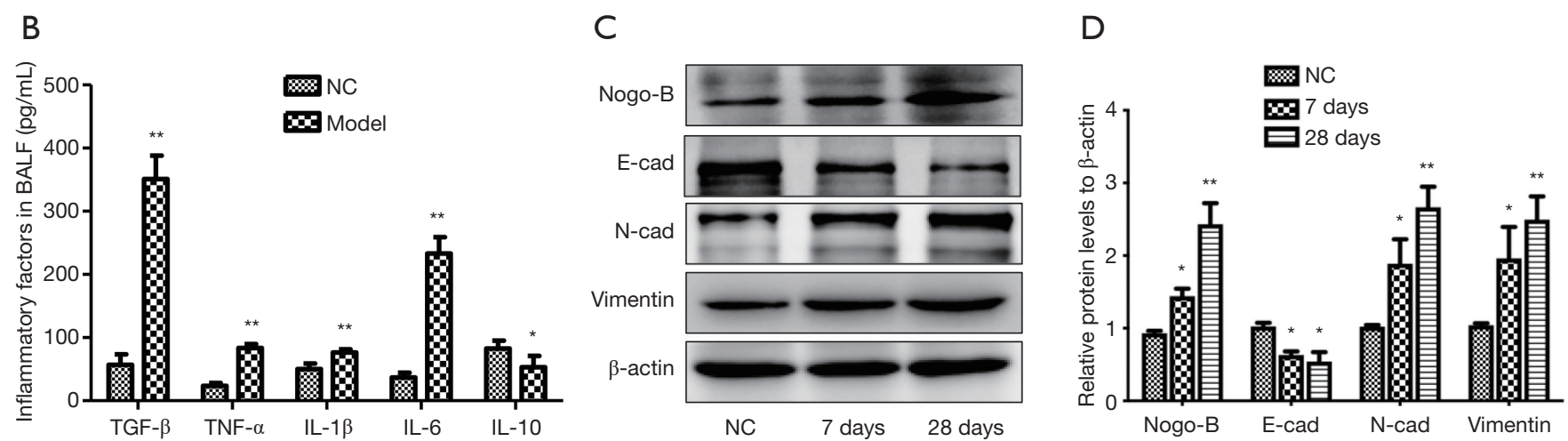

Figure 1 Nogo-B expression was up-regulated in a mouse model of BLM-mediated pulmonary fibrosis. (A) Representative images of Masson staining and Nogo-B immunohistochemical staining control lung tissues and lung tissues at the $28^{\text {th }}$ day from a pulmonary fibrosis mouse model at a magnification of $\times 200$ and then representative fields were chosen for presentation. NC, normal control. Scale bars show $50 \mu \mathrm{m}$. (B) Levels of TGF- $\beta$, TNF- $\alpha$, IL-1 $\beta$, IL-6 and IL-10 in bronchoalveolar lavage fluid (BALF) was detected with enzyme-linked immunosorbent assay (ELISA). (C,D) Western blotting analysis for Nogo-B expression in lung tissues. Error bars indicate standard error of mean (SEM); $\mathrm{n}=3$. * indicates $\mathrm{P}<0.05$ vs. control groups; **, indicates $\mathrm{P}<0.01$ vs. control groups. IHC, immunohistochemistry.

EMT of lung fibrosis.

\section{Nogo-B knockdown significantly attenuated lung fibrogenesis}

To further determine the effect of Nogo-B on the lung fibrogenesis, we observed the BLM-induced lung fibrogenesis in wild-type mice and Nogo-B knockout mice. It was found that the late (the $7^{\text {th }}$ and $28^{\text {th }}$ day) fibrotic response was significantly reduced in knockout mice compared with wild-type mice (Figure $2 A$ ); meanwhile, levels of pro-inflammatory cytokines including TGF- $\beta$, TNF- $\alpha$, IL-1 $\beta$, IL- 6 were significantly suppressed (all $\mathrm{P}<0.01)$ while anti-inflammatory factor IL-10 was upregulated $(\mathrm{P}<0.05)$ in BALF (Figure $2 B)$.

To investigate the alteration of alveolar epithelial cells in wild-type mice and Nogo-B knockout mice, we detected apoptosis-related markers-Bcl-2 and cleaved caspase- 3 in purified AECII and found that the expression of Bcl-2 was 1.5 times higher in Nogo- $\mathrm{B}^{-/-}$mice than that in control one and the level of cleaved caspase- 3 dropped to less than half of the BLM-treated group (Figure 2C,D), suggesting the inhibition of apoptosis in alveolar epithelial cells when Nogo-B was absent. As mentioned above, some researches have indicated the relationship between EMT and ERS; hence, we examined the expressions of ERS-related key sensors including PERK, IRE1 $\alpha$, ATF6 as well as E-cadherin and $\mathrm{N}$-cadherin, vimentin in purified AECIIs from wildtype mice and Nogo-B knockout mice and observed that compared with AECIIs in wild-type mice, AECIIs in Nogo-B knockout mice presented the significant reduction of only PERK expression among the three ERS-related sensors, as well as the promotion of E-cadherin expression and the inhibition of $\mathrm{N}$-cadherin and vimentin expressions. These results suggested Nogo-B may influence the ERS or EMT in AECIIs (Figure 2E,F). 
A

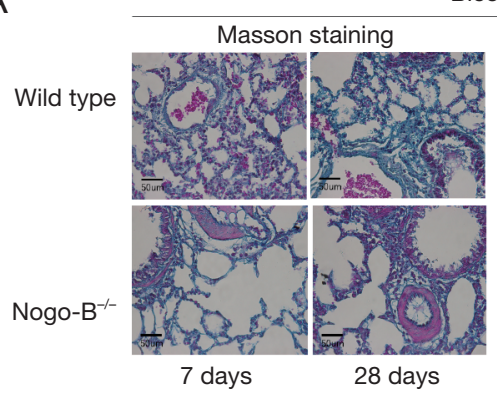

Bleomycin

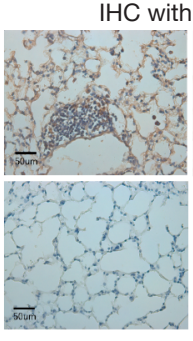

7 days

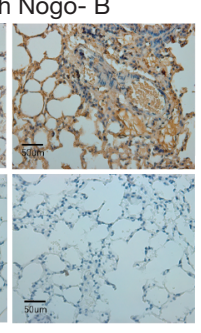

28 days

E

C
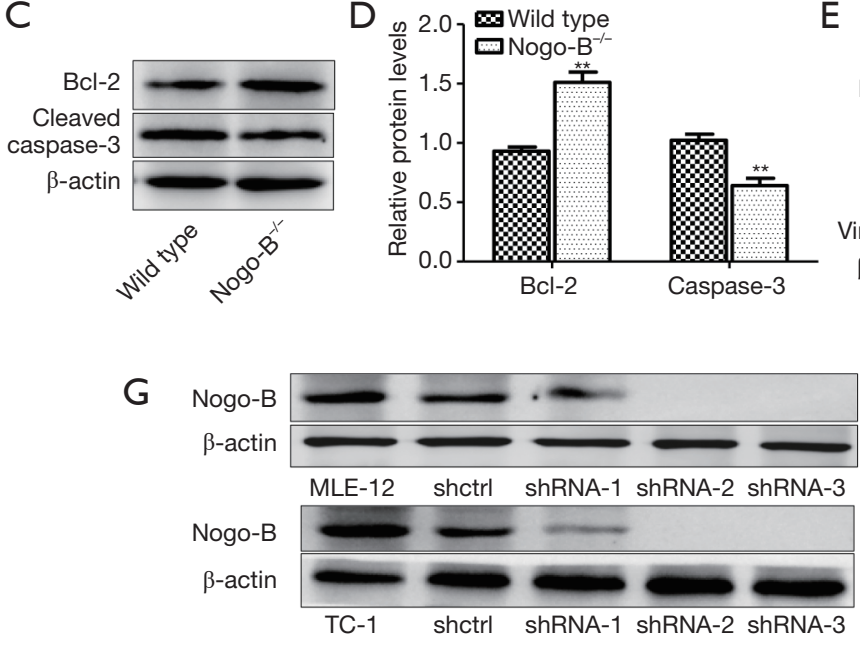

I
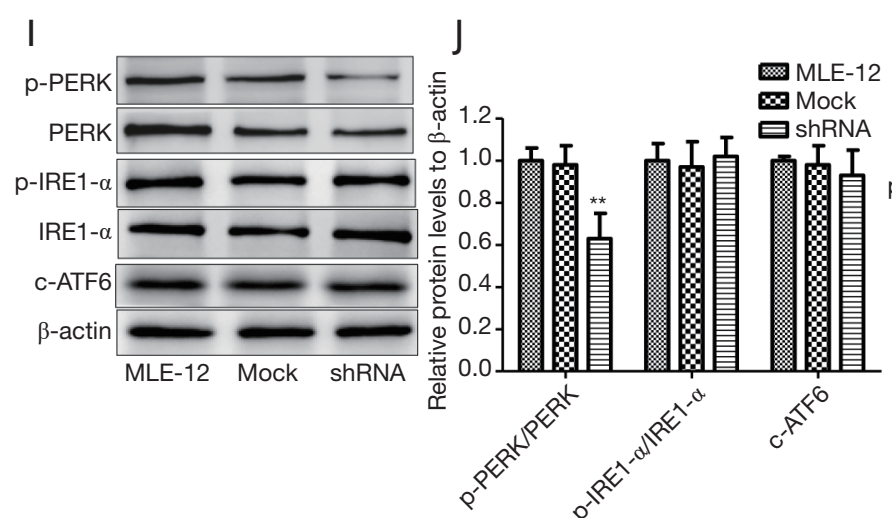

B

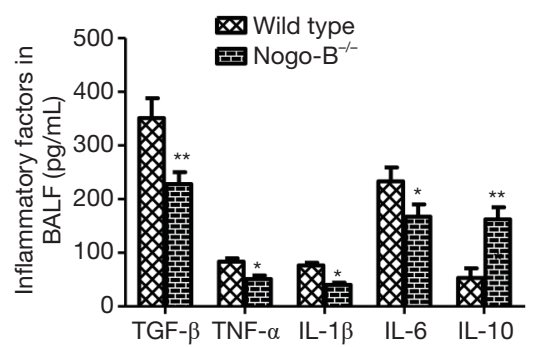

$\mathrm{F}$

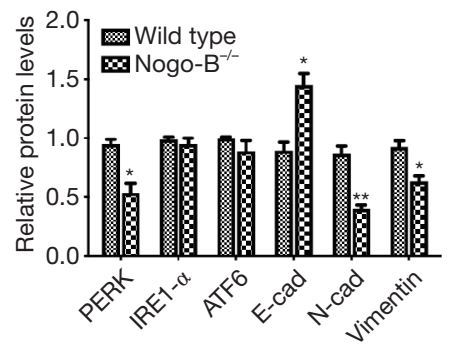

$\mathrm{H}$

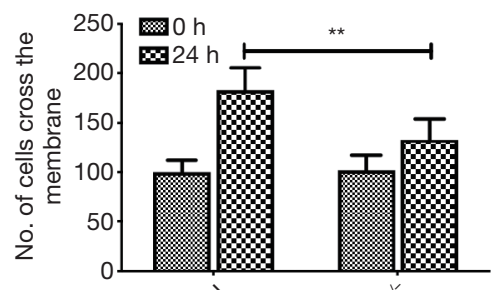

$\mathrm{K}$

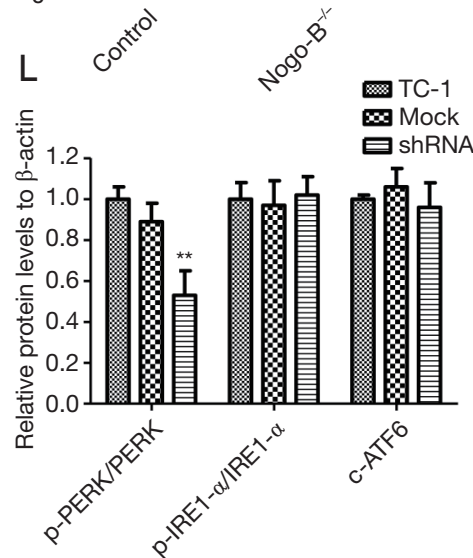

Figure 2 Nogo-B knockdown significantly attenuated lung fibrogenesis. (A) Representative images of Masson staining and Nogo-B immunohistochemical staining control lung tissue and lung tissue at the $7^{\text {th }}$ and $28^{\text {th }}$ day during constructing a pulmonary fibrosis model of Nogo-B knockout mice at a magnification of $\times 200$ for presentation. NC, normal control. Scale bars show $50 \mu \mathrm{m}$. (B) Levels of TGF- $\beta$, TNF- $\alpha$, IL-1 $\beta$, IL-6 and IL-10 in bronchoalveolar lavage fluid (BALF) of Nogo-B knockout mice were detected with enzyme-linked immunosorbent assay (ELISA). (C,D) Western blotting analysis for expressions of Bcl-2 and cleaved caspase-3 in purified AECII from wildtype mice and Nogo-B knockout mice. (E,F) Western blotting analysis for expressions of PERK, IRE1 $\alpha$, ATF6 as well as E-cadherin and $\mathrm{N}$-cadherin in purified AECIIs from wild-type mice. (G) the expression of Nogo-B in two murine lung epithelial cell lines-MLE-12 and TC-1 JHU-1 was knocked down based on three target sequences. (H) Transwell analysis for migration in murine lung epithelial cells. (I,J,K,L) Western blotting analysis for expressions of endoplasmic reticulum stress (ERS)-related markers including p-PERK/PERK, p-IRE1 $\alpha /$ IRE1 $\alpha$ and cleaved ATF6 (c-ATF6). Error bars indicate standard error of mean (SEM); $\mathrm{n}=3 .{ }^{*}$ indicates $\mathrm{P}<0.05$ vs. control groups; **, indicates $\mathrm{P}<0.01$ vs. control groups. IHC, immunohistochemistry. 
To further determine the effect of Nogo-B on the activity of ERS-related pathways, we knocked down effectively the expression of Nogo-B in two murine lung epithelial cell lines-MLE-12 and TC-1 JHU-1 based on three target sequences and chose shRNA-3 for subsequent experiments (Figure 2G). After Nogo-B knockdown, the migration ability of MLE-12 was inhibited (Figure 2H); meanwhile both in MLE-12 and in TC-1 JHU-1, the ratio of phosphor-PERK related to total PERK was significantly decreased while no much difference showed in the levels of phosphor-IRE1 $\alpha /$ IRE1 $\alpha$ and cleaved ATF6 (c-ATF6) (Figure 2I, f,K,L). The result suggested Nogo-B may regulate the activation of PERK branch but not the activation of IRE1 $\alpha$ or ATF6 branches of ERS pathway in murine lung epithelial cells.

\section{Inhibition of Nogo-B in fibrogenesis was associated with the PERK branch of ERS patbway}

To further reveal the detailed effect of Nogo-B on lung fibrogenesis, we firstly simulated the fibrogenesis of lung epithelial cells through treating the MLE-12 or TC-1 JHU-1 in vitro with TGF- $\beta 1$ (10 ng/mL for $24 \mathrm{~h})$. The results showed that in MLE-12 and TC-1 JHU-1 cells, TGF- $\beta 1$ induced the increase of phosphor-PERK/PERK but not phosphor-IRE1 $\alpha /$ IRE $1 \alpha$ and c-ATF6; however, when Nogo-B knockdown, such an induced effect of TGF- $\beta 1$ on phosphor-PERK/PERK was not significant (Figure $3 A, B, C, D$ ). It suggested a necessity of Nogo-B in the regulation of PERK branch activation of ERS pathway. Moreover, TGF- $\beta 1$ also enhanced the migration of lung epithelial cells; in contrast, Nogo-B knockdown suppressed the enhancement of TGF- $\beta 1$-induced migration in lung epithelial cells (Figure $3 E, F$ ). In addition, the expressions of some EMT or fibrogenesis-related markers including tight junction protein ZO-1, type II alveolar surfactant protein $\mathrm{C}$ (SPC), $\alpha$-SMA and MMP4 were also investigated. It was found that TGF- $\beta 1$ resulted in the reduction of ZO-1 and SPC as well as the elevation of $\alpha$-SMA and MMP4; however, after Nogo-B knockdown, such phenomenon were not observed in both cell lines (Figure 3G,H,I,7). The result suggested the inhibition of Nogo-B retarded fibrogenesis in lung epithelial cells via affecting PERK activation and EMT signaling.

\section{Blockade of PERK activation inbibited lung fibrogenesis}

To further investigate the potential effects of Nogo-B via regulating PERK activation on lung fibrogenesis, we achieved lentivirus-mediated Nogo-B overexpression in lung epithelial cells; then examined the expressions of PERK and its downstream transcript factor ATF4. It was found that both PERK and ATF4 were increased after Nogo-B overexpression (Figure $4 A, B, C, D$ ). Moreover, to demonstrate whether PERK is involved in Nogo-Bmediated EMT or not, we downregulated the expression of PERK in lung epithelial cells with Nogo-B overexpression and found, after PERK knockdown, Nogo-B-induced the upregulation of E-cadherin and the downregulation of $\mathrm{N}$-cadherin and vimentin were significantly inhibited (Figure $4 E, F, G, H$ ). These findings furtherly indicated the involvement of Nogo-B in lung fibrogenesis through regulating PERK branch of ERS pathway; at the same time, it suggested the possibility of PERK blockade in treating the lung fibrosis. Herein, we applied the PERK blockade (ISRIB; $0.25 \mathrm{mg} / \mathrm{kg}$ ) to treat the model mice and found the gradual improvement of lung fibrosis content as time consists (Figure $4 I$ ) and the EMT process of purified AECIIs was partly attenuated (Figure $4 \mathcal{F}, K$ ); meanwhile the levels of TGF- $\beta$ and pro-inflammatory cytokines including TNF- $\alpha$, IL- $1 \beta$, IL- 6 were declined and the level of IL-10 was reversed in BALF (Figure 4L). These findings suggested a potential utility of PERK blockade in treating lung fibrosis.

\section{Discussion}

At present, as a common pulmonary interstitial disease, IPF's pathogenesis is still unclear (17). Accumulating studies have demonstrated that EMT plays an important role in the development of IPF-related fibrogenesis; epithelial cells experienced EMT and transformed into fibroblasts, accounting for about $50 \%$ of the total number of fibroblasts $(5,18)$. As the number of fibroblasts increased, collagen and extracellular matrix gradually accumulated. In the study, the EMT alteration of alveolar epithelial cells and the accumulation of collagen and extracellular matrix were observed in the model mice, which can clearly determine the degree of tissue fibrosis. Of note, in our study, it was also found that the lung tissue of Nogo-B knockout mice posed a significant inhibition of pulmonary interstitial fibrosis, compared to wild type mice. It suggested that Nogo-B knockout can interfere with the fibrogenesis of lung tissue.

As a member of RTN4 family, Nogo-B plays an important role in inflammation and tissue repair (19-21). Our study also confirmed that during BLM-induced lung 
A

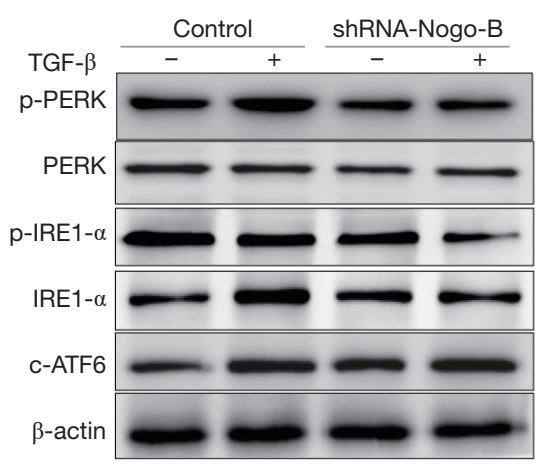

C

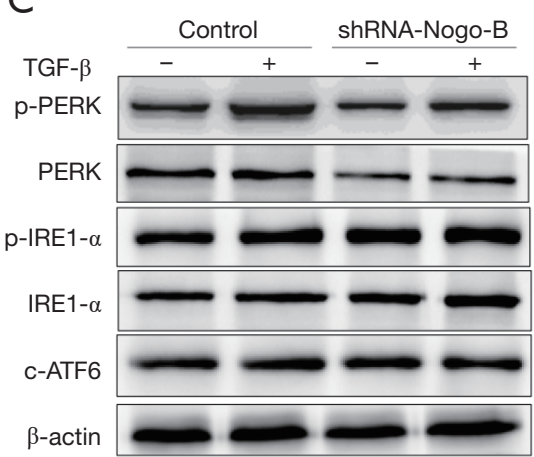

G
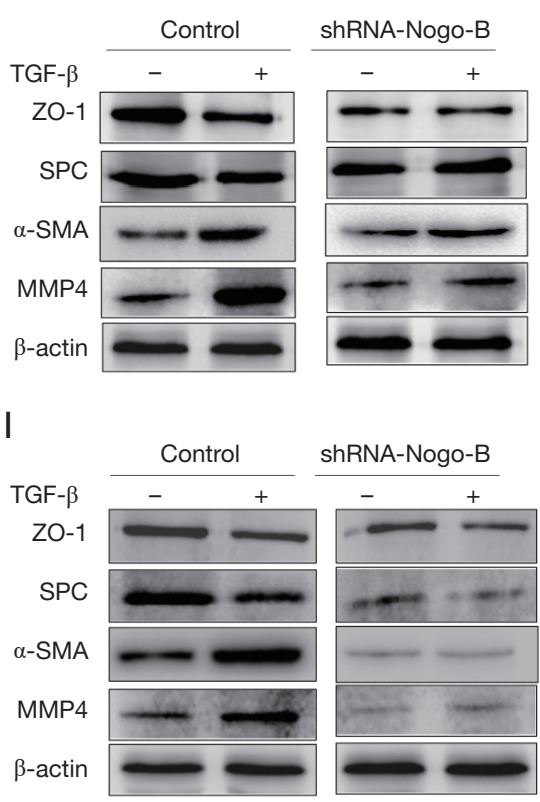

B

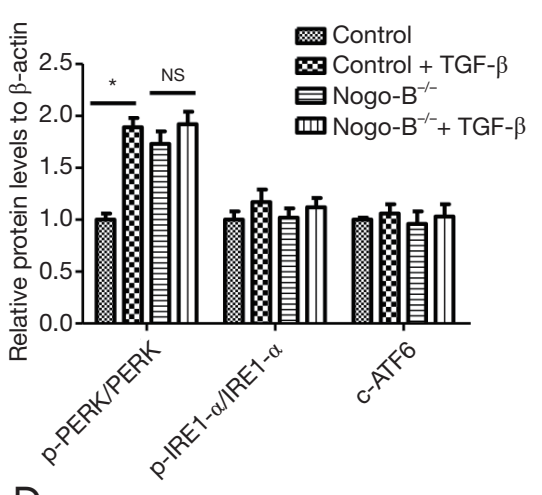

D

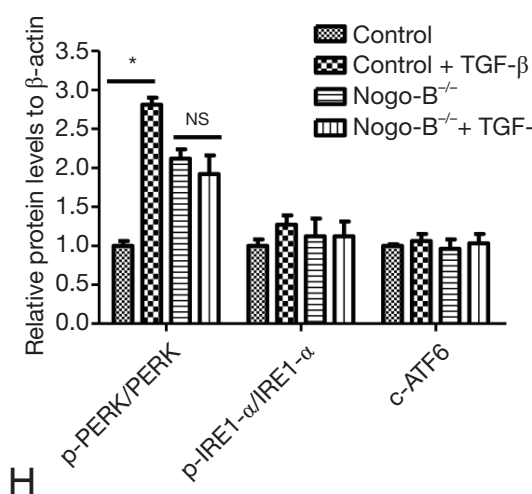

$\mathrm{F}$
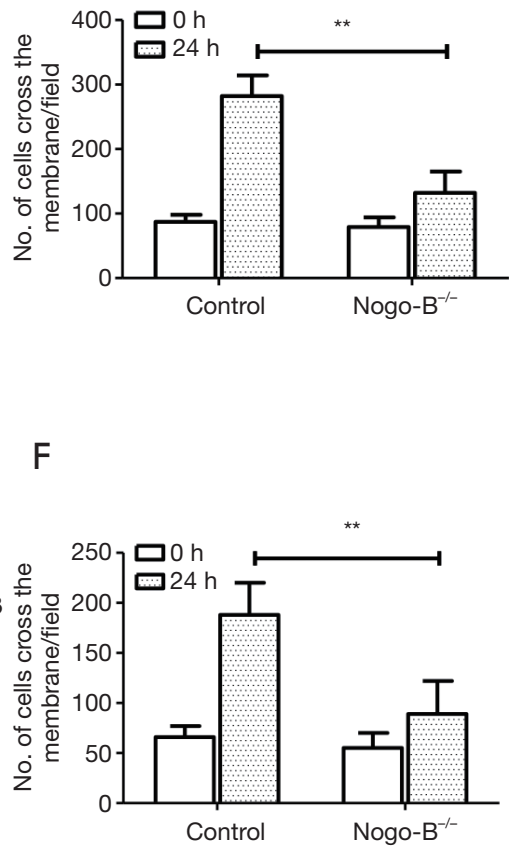

Figure 3 Effects of Nogo-B on the endoplasmic reticulum stress (ERS) pathway during lung fibrogenesis. (A,B,C,D) Western blotting analysis for ERS sensors in Nogo-B knockdown lung epithelial cells MLE-12 and TC-1 JHU-1 treated with TGF- $\beta 1$ (10 ng/mL for 24 h). (E,F) Transwell analysis for migration in Nogo-B knockdown lung epithelial cells MLE-12 and TC-1 JHU-1 treated with TGF- $\beta 1$. (G,H,I,J) Western blotting analysis for some epithelial-mesenchymal transition (EMT) or fibrogenesis-related markers including tight junction protein ZO-1, type II alveolar surfactant protein C (SPC), $\alpha$-SMA and MMP4 in Nogo-B knockdown lung epithelial cells MLE-12 and TC-1 JHU-1 treated with TGF- $\beta 1$. Error bars indicate standard error of mean (SEM); $n=3$. * indicates $\mathrm{P}<0.05$; **, indicates $\mathrm{P}<0.01$. (A,B,E,G,H): MLE-12; (C,D,F,I, J): in TC-1 JHU-1. 


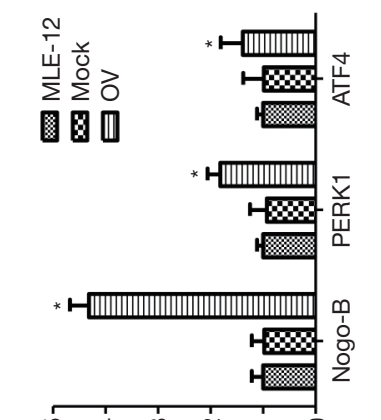

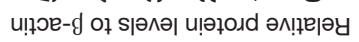

$\square$

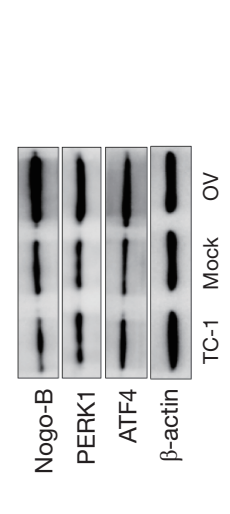

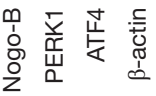

U

$\checkmark$

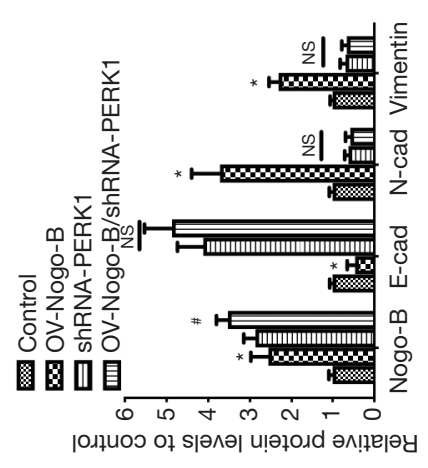

I

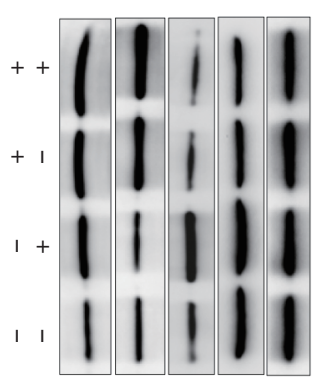

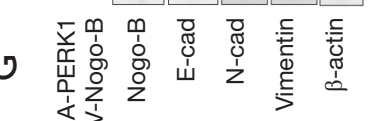
$\sum_{\substack{x \\ \frac{1}{n}}}^{+}$

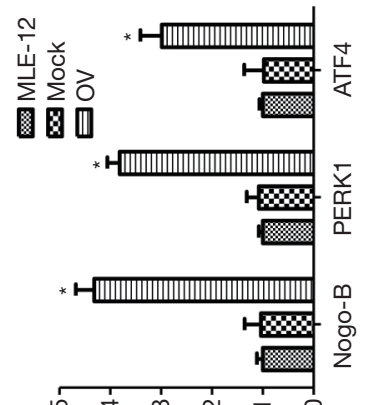

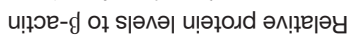

$\infty$

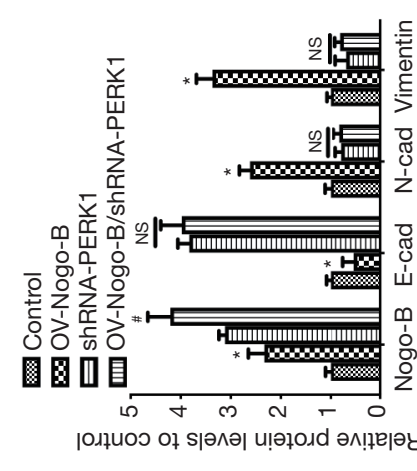

$\varangle$

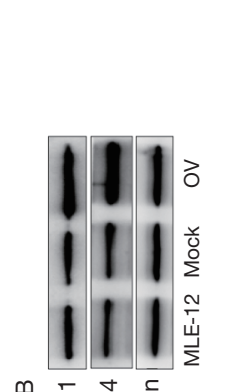

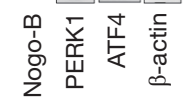

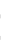

ᄂ

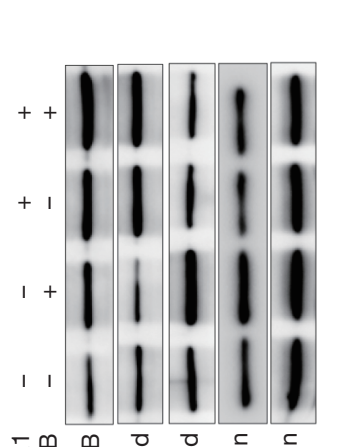

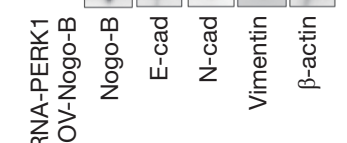

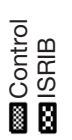

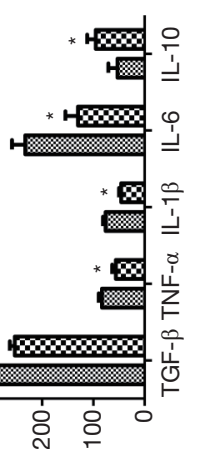

(7m/6d) J7f

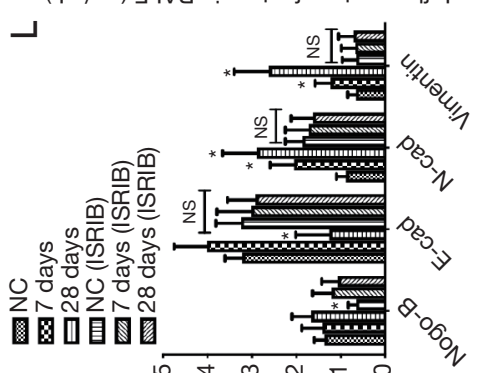

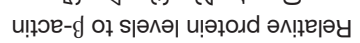

$\underline{v}$
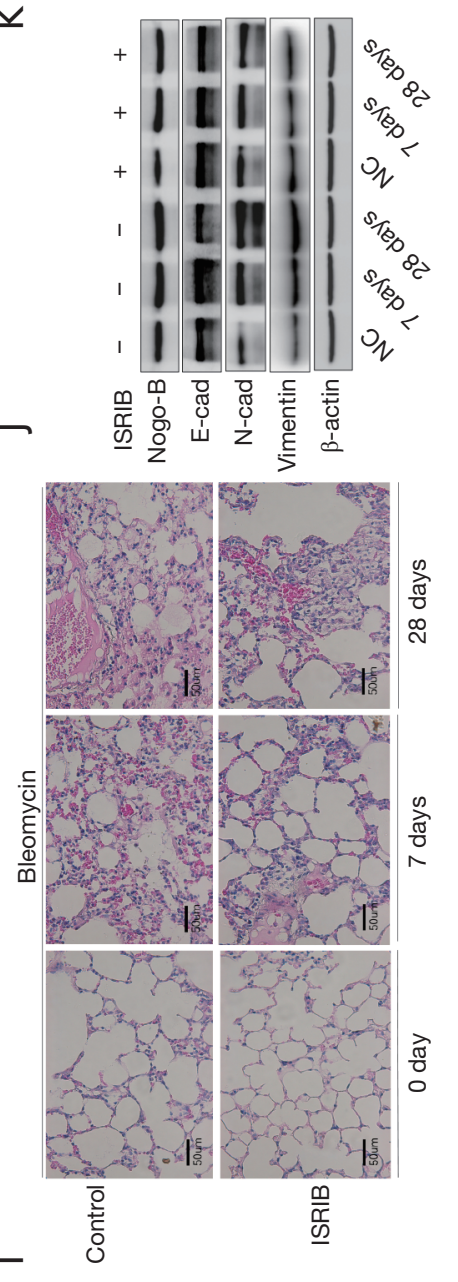

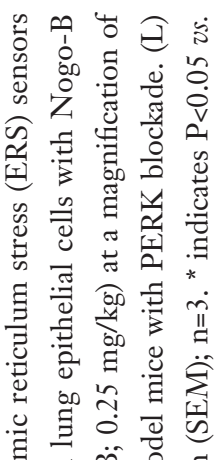

च $\ddot{\theta}$

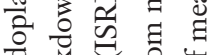

ป प

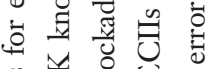

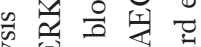

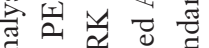

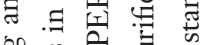

.

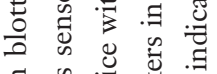

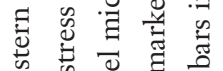

उ क च है

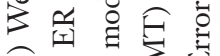

ใิ

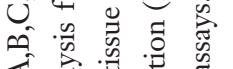

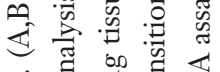

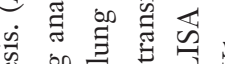

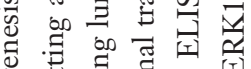

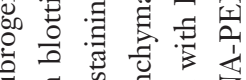

I

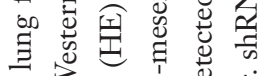

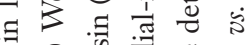

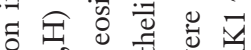

包菏

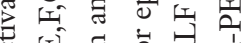

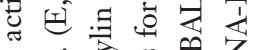

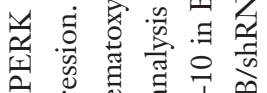

च च

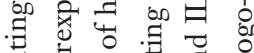

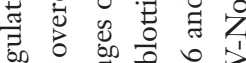

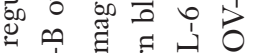

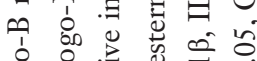

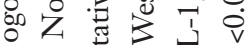

Z 至

पे के

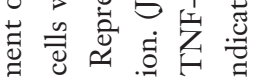

ป

疍

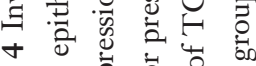

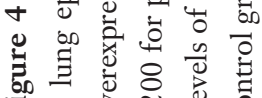


fibrogenesis, the expression of Nogo-B increased with the occurrence of fibrosis; the result indicated that Nogo-B was involved in the occurrence of inflammatory and fibrotic response. Furthermore, EMT in alveolar epithelial cells in the Nogo-B knockout group was inhibited, compared with that in the wild-type group, which means that Nogo-B may affect fibrogenesis through the EMT response (13). However, what's the molecular mechanism of Nogo-B affecting EMT in lung fibrogenesis? This question requires us to verify through further experiments.

Because Nogo-B is widely expressed in ER, it functions as a crucial protein for maintaining the ER-mitochondria unit (22) and plays an important role in protein folding, ER homeostasis, and lipid biosynthesis (23). It was recently reported Nogo-B can regulate the distance between ER and mitochondria when stress exists (24). Moreover, recent research demonstrated that upregulating Nogo-B expression helps to inhibit the proliferation of pulmonary artery smooth muscle cells, oxidative stress and ER stress (25). Meanwhile, Nogo-B can also modify polarization of Kupffer cells in liver (26) and its regulation on the TGF- $\beta$-smad2 signaling may be an important way to modulate liver tissue fibrosis repairment (11). In addition, Nogo-B can promote hepatocyte growth factor (HGF)-induced hepatocyte proliferation via IL-6/STAT3 signaling pathway and reduce the inhibitory effect of TGF- $\beta$ on proliferation (27). However, the relationship between Nogo-B and ERS in lung fibrogenesis was not fully studied. ERS induces the transduction of three independent ERS signaling pathways via three transmembrane proteins PERK, ATF6 and IRE1 on the endoplasmic reticulum membrane (28). In the study, Nogo-B knockdown only downregulated significantly expression of PERK among the three ERS-related sensors, with no significant effects on expressions of IRE $1 \alpha$ and ATF6; it suggested Nogo-B may regulate the activation of PERK branch of ERS pathway in murine lung epithelial cells. Mechanistically, Nogo-B knockdown inhibited the increase of TGF- $\beta 1$-induced EMT or fibrogenesis-related markers expression; meanwhile suppressed the upregulation of TGF$\beta 1$-induced PERK branch of ERS pathway. These results suggested Nogo-B was necessary for activation of PERK branch of ERS pathway in lung fibrosis. All these findings indicated ERS and EMT may play an important role in the occurrence and development of pulmonary fibrosis. Hence, unravelling the internal mechanism is greatly significant for further understanding the mechanism of pulmonary fibrosis or exploring effective strategies to prevent pulmonary fibrosis. As is now well known, existing drugs can only relieve or delay the symptoms of IPF and cannot fundamentally cure the disease (29). Currently, drug research and development institutions in many countries have focused on the research and development of IPF therapeutic drugs, but the progress is limited (30). In the study, we further explored the potential utility of PERK blockade in treating the lung fibrosis and observed the gradual improvement of lung fibrosis content as time consists, suggesting a potential efficacy of PERK blockade in treating lung fibrosis.

\section{Conclusions}

There are several limitations in our study. We had not elucidated the exhaustive molecular mechanism for explaining the regulation of Nogo-B in the expressions of ERS- and EMT-markers at transcriptional or posttranscriptional levels, which consequently leading to lung fibrogenesis. Moreover, exact role of Nogo-B in constructing the association between ERS and EMT in lung fibrosis is still to be verified. In addition, the current study is purely a murine study without human samples. Nevertheless, from the available data, we are able to reveal that Nogo-B functions as a mediator between EMT and ERS in promoting lung fibrogenesis, from mouse model in vivo and cell lines model in vitro; Nogo-B can be considered a potential therapeutic target for the treatment of IPF. Of course, more detailed studies in human samples or disease models are needed to determine the feasibility and therapeutic benefit of targeting Nogo-B in pulmonary fibrotic diseases in the future.

\section{Acknowledgments}

We wish to thank Ms. Bo Wang for her assistance in animal experiment and Mr. Peter Wong for language editing.

Funding: This work was supported by the National Natural Science Foundation of China (Grant No. 81700069 and 81870064) and the Clinical Research Project supported by Beijing Municipal Science \& Technology Commission (Z181100001718024).

\section{Footnote}

Reporting Checklist: The authors have completed the ARRIVE reporting checklist. Available at http://dx.doi. org/10.21037/atm-20-6143

Data Sharing Statement: Available at http://dx.doi. 
org/10.21037/atm-20-6143

Peer Review File: Available at http://dx.doi.org/10.21037/ atm-20-6143

Conflicts of Interest: All authors have completed the ICMJE uniform disclosure form (available at http://dx.doi. org/10.21037/atm-20-6143). The authors have no conflicts of interest to declare.

Ethical Statement: The authors are accountable for all aspects of the work in ensuring that questions related to the accuracy or integrity of any part of the work are appropriately investigated and resolved. Experiments were performed under a project license (No. 2020-097) granted by the Ethics Committee of the Seven Medical Center of PLA General Hospital, in compliance with institutional guidelines for the care and use of animals.

Open Access Statement: This is an Open Access article distributed in accordance with the Creative Commons Attribution-NonCommercial-NoDerivs 4.0 International License (CC BY-NC-ND 4.0), which permits the noncommercial replication and distribution of the article with the strict proviso that no changes or edits are made and the original work is properly cited (including links to both the formal publication through the relevant DOI and the license). See: https://creativecommons.org/licenses/by-nc-nd/4.0/.

\section{References}

1. Richeldi L, Collard HR, Jones MG. Idiopathic pulmonary fibrosis. Lancet 2017;389:1941-52.

2. Merkt W, Bueno M, Mora AL, et al. Senotherapeutics: targeting senescence in idiopathic pulmonary fibrosis. Semin Cell Dev Biol 2020;101:104-10.

3. Upagupta C, Shimbori C, Alsilmi R, et al. Matrix abnormalities in pulmonary fibrosis. Eur Respir Rev 2018;27:180033.

4. Korfei M, Skwarna S, Henneke I, et al. Aberrant expression and activity of histone deacetylases in sporadic idiopathic pulmonary fibrosis. Thorax 2015;70:1022-32.

5. Kage H, Borok Z. EMT and interstitial lung disease: a mysterious relationship. Curr Opin Pulm Med 2012;18:517-23.

6. Miao C, Xiong Y, Zhang G, et al. MicroRNAs in idiopathic pulmonary fibrosis, new research progress and their pathophysiological implication. Exp Lung Res
2018;44:178-90.

7. Zhang L, Wang Y, Pandupuspitasari NS, et al.

Endoplasmic reticulum stress, a new wrestler, in the pathogenesis of idiopathic pulmonary fibrosis. Am J Transl Res 2017;9:722-35.

8. Li YK, Xie YJ, Wu DC, et al. Nogo B receptor in relevant carcinoma: current achievements, challenges and aims (Review). Int J Oncol 2018;53:1827-35.

9. Cantalupo A, Zhang Y, Kothiya M, et al. Nogo-B regulates endothelial sphingolipid homeostasis to control vascular function and blood pressure. Nat Med 2015;21:1028-37.

10. Kimura T, Endo S, Inui M, et al. Endoplasmic Protein Nogo-B (RTN4-B) Interacts with GRAMD4 and Regulates TLR9-Mediated Innate Immune Responses. J Immunol 2015;194:5426-36.

11. Zhang D, Utsumi T, Huang HC, et al. Reticulon $4 \mathrm{~B}$ (Nogo-B) is a novel regulator of hepatic fibrosis. Hepatology 2011;53:1306-15.

12. Men R, Wen M, Dan X, et al. Nogo-B: A potential indicator for hepatic cirrhosis and regulator in hepatic stellate cell activation. Hepatol Res 2015;45:113-22.

13. Xiong Y, Zhang J, Shi L, et al. NOGO-B promotes EMT in lung fibrosis via MMP14 mediates free TGF-beta1 formation. Oncotarget 2017;8:71024-37

14. Zhou Y, Li P, Duan JX, et al. Aucubin alleviates bleomycin-induced pulmonary fibrosis in a mouse model. Inflammation 2017;40:2062-73.

15. Fu Q, Tao Y, Piao H, et al. Trophoblasts and decidual stromal cells regulate decidual NK cell functions via interaction between collagen and LAIR-1. Am J Reprod Immunol 2014;71:368-78.

16. Chuquimia OD, Petursdottir DH, Rahman MJ, et al. The role of alveolar epithelial cells in initiating and shaping pulmonary immune responses: communication between innate and adaptive immune systems. PLoS One 2012;7:e32125.

17. Olson AL, Gifford AH, Inase N, et al. The epidemiology of idiopathic pulmonary fibrosis and interstitial lung diseases at risk of a progressive-fibrosing phenotype. Eur Respir Rev 2018;27:180077.

18. Coward WR, Saini G, Jenkins G. The pathogenesis of idiopathic pulmonary fibrosis. Ther Adv Respir Dis 2010;4:367-88.

19. Long SL, Li YK, Xie YJ, et al. Neurite outgrowth inhibitor b receptor: a versatile receptor with multiple functions and actions. DNA Cell Biol 2017;36:1142-50.

20. Zhu Y, Tong Q, Ye J, et al. Nogo-B facilitates LPSmediated immune responses by up-regulation of TLR4- 


\section{Page 12 of 12}

signaling in macrophage RAW264.7. Cell Physiol Biochem 2017;41:274-85.

21. Xu W, Zhu Y, Ning Y, et al. Nogo-B protects mice against lipopolysaccharide-induced acute lung injury. Sci Rep 2015;5:12061.

22. Sutendra G, Dromparis P, Wright P, et al. The role of Nogo and the mitochondria-endoplasmic reticulum unit in pulmonary hypertension. Sci Transl Med 2011;3:88ra55.

23. Harrison KD, Park EJ, Gao N, et al. Nogo-B receptor is necessary for cellular dolichol biosynthesis and protein N-glycosylation. EMBO J 2011;30:2490-500.

24. Yang YD, Li MM, Xu G, et al. Nogo-B receptor directs mitochondria-associated membranes to regulate vascular smooth muscle cell proliferation. Int J Mol Sci 2019;20:2319.

25. Tadokoro KS, Rana U, Jing X, et al. Nogo-B receptor modulates pulmonary artery smooth muscle cell function in developing lungs. Am J Respir Cell Mol Biol

Cite this article as: Zhu Y, Yang M, Li XH, Xu WJ, Gao W, Chen YH, Li JD, Li Q. Nogo-B promotes epithelialmesenchymal transition in lung fibrosis via PERK branch of the endoplasmic reticulum stress pathway. Ann Transl Med 2021;9(7):563. doi: 10.21037/atm-20-6143

\section{Zhu et al. Nogo-B and PERK activation in lung fibrogenesis}

2016;54:892-900.

26. Park JK, Shao M, Kim MY, et al. An endoplasmic reticulum protein, Nogo-B, facilitates alcoholic liver disease through regulation of kupffer cell polarization. Hepatology 2017;65:1720-34.

27. Gao L, Utsumi T, Tashiro K, et al. Reticulon 4B (Nogo-B) facilitates hepatocyte proliferation and liver regeneration in mice. Hepatology 2013;57:1992-2003.

28. Hetz C, Saxena S. ER stress and the unfolded protein response in neurodegeneration. Nat Rev Neurol 2017;13:477-91.

29. Aiello M, Bertorelli G, Bocchino M, et al. The earlier, the better: impact of early diagnosis on clinical outcome in idiopathic pulmonary fibrosis. Pulm Pharmacol Ther 2017;44:7-15.

30. Martinez FJ, Collard HR, Pardo A, et al. Idiopathic pulmonary fibrosis. Nat Rev Dis Primers 2017;3:17074. 